$\xi=-1$

\title{
Implementation of master-slave method on multiprocessor-based embedded system: case study on mobile robot
}

\author{
Agusma Wajiansyah ${ }^{1}$, Hari Purwadi ${ }^{2}$, Asrina Astagani ${ }^{3}$, Supriadi Supriadi ${ }^{4 *}$ \\ 1,2,3,4 Department of Information Technology, State Polytechnic of Samarinda, East Kalimantan, Indonesia \\ *Corresponding author E-mail: supriadi.polnes@gmail.com
}

\begin{abstract}
In this research the master-slave method implemented on an embedded system using 3 processor applied to the mobile robot, to know the speed of program execution of robot. As a comparison is also used a robot with an embedded system based on single processor. From the experimental results, by applying the slave master method obtained the execution time of $546,5 \mu$ s and the number of iteration 1079 , while for single processor-based system obtained execution time average $67828 \mu$ s and the amount of iteration average 147 times. Where the number of iterations is obtained by running the robot for $10 \mathrm{~s}$. From this experiment, it can be concluded that there is a performance increase of $7.3 \%$ when compared to embedded systems based on single processor.
\end{abstract}

Keywords: multiprocessor; master-slave; time of program execution.

\section{Introduction}

The use of mobile robot looks increasing in industrial or household. in the industry many things can be done such as cleaning floors, loading and unloading in factories, transporting samples from one location to another and so forth. With various types of robot development, also used for domestic workers. This is done because of its flexibility, small size, and low cost. Whatever type of robot definitely needs a computer device that will run it. This computer is also called embedded system.

The mobile robot is a robot with the ability to move position in an environment using its own resources. One type of mobile robot is a wheeled robot. The wheeled robot is basically divided into two types, namely holonomic that can move all directions without changing the orientation and non-holonomic that moves in a direction by turning or changing orientation. Wheels on holonomic are mecanum wheel or Omni wheel and on non-holonomic using the conventional wheel. In a wheeled robot if using 2 wheels drive is called $2 \mathrm{WD}$ or using 4 wheels drive is called $4 \mathrm{WD}$. To drive the robot, an embedded device system is designed specifically for the robot.

According to [1][10][11][12], embedded systems is a combination of hardware and software to respond to the surrounding events within the specified limits. Embedded systems can consist of a small computer with microprocessor or microcontroller and supporting devices. In the robot application, the microcontroller will perform the procedure in a sequential manner, i.e. from the process of acquiring and processing (perception) data from the sensor, run robot algorithm, and specify the action that must be done. From the procedure, the process of data acquisition of the sensor is time-consuming, how much time delay very greatly depending on the type of sensor used. For example, for distance sensor based on ultrasonic-wave with a maximum reading distance of $300 \mathrm{~cm}$, a maximum delay of $17.4 \mathrm{~ms}$. This can be a constraint, because according to [1] an embedded system must react quickly to environmental changes and be able to respond to events in real time with small delay

One method that can be used to speed up the control process is by adding the number of processors. All processors on this system will communicate with the Master-Slave method. Master-slave is a communication model where one device or process has direct control over one or more other devices. Or in other words, there is only one device or process that is active, while others are passive. This method is commonly used as in [4][5][6][7].

According to [3] this is one method that can be used on multiprocessor systems.

\section{Experimental Method}

To support this research used tools such as a mobile robot with 4 wheels drive and equipped with 4 pieces of ultrasonic wave based distance sensors mounted on each side, and embedded system design using a microcontroller. The embedded systems for wheeled robots in this research are described in Figure 1 for systems using single processor and Figure 2 for systems using master-slave method.

The multiprocessor system used 3 pieces of microcontroller, one of them serves as a master which will run the main program for the robot system. Another microcontroller serves as a slave. Each slave serves as a data processor of the acquisition process and the actuator controller. This is shown in Figure 2. All microcontrollers work with clock speeds of $16 \mathrm{MHz}$ and communicate using an I2C interface with a clock frequency of $100 \mathrm{KHz}$. 


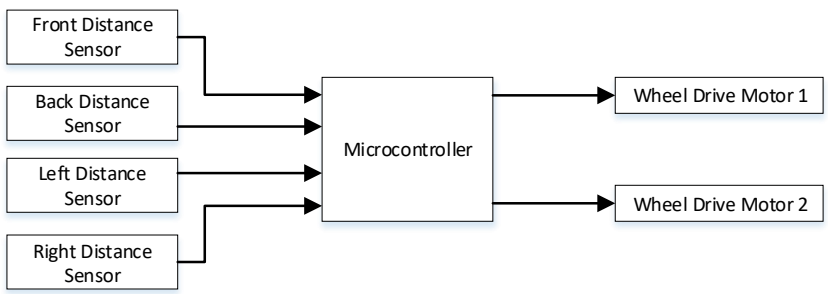

Fig. 1: Block Diagram of Embedded System on Mobile Robot using a single microcontroller

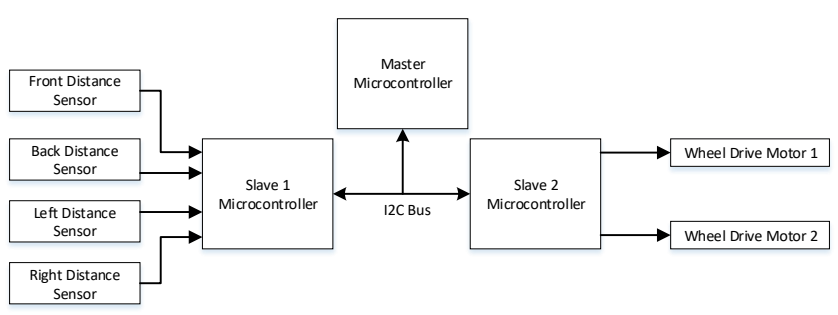

Fig. 2: Block Diagram of Embedded System on Mobile Robot using master-slave microcontroller.

There To interact between master and slave begins by master, which will start communication by sending a message to slave. When the slave receives the message, proceed with translating it The result is an immediate task performed by the slave. Once completed the slave will inform the master again, or send the specified data if the message from the master is a data request. This interaction system is also known as inter-process communication The Sequential process on master slaver as shown in Figure 4.

From figure 4, 'motor control' is the main process run by slave 2 . In this process will generate PWM signal which will be given to the motor driver. The PWM update value is given from the master through $\mathrm{I} 2 \mathrm{C}$ communication. The process of receiving data using interrupt driven. When no data is sent then the microcontroller is idle.

While in slave 1 'acquisition and perception' is the main process. This process aims to acquire data from all sensors and perception them. The result will be stored in memory. This process runs with a time interval of $100 \mathrm{~ms}$, so the data in memory will be updated at that time interval. This data will be send when there is a request from the master. Receipt of requests and data sending is done by interrupt driven.

Figure 3 is a planned procedure for a single processor system. Figure 5 is a state diagram for the procedure on the slave with data processing function of the acquisition process, while on Figure 6 is the state diagram for the procedure on the slave that controls the actuator.

\begin{tabular}{|l|l|l|}
\hline Acquisition And Perception & Robot Fuction algoritma & Action \\
\hline
\end{tabular}

Fig. 3: Sequential process on single processor.

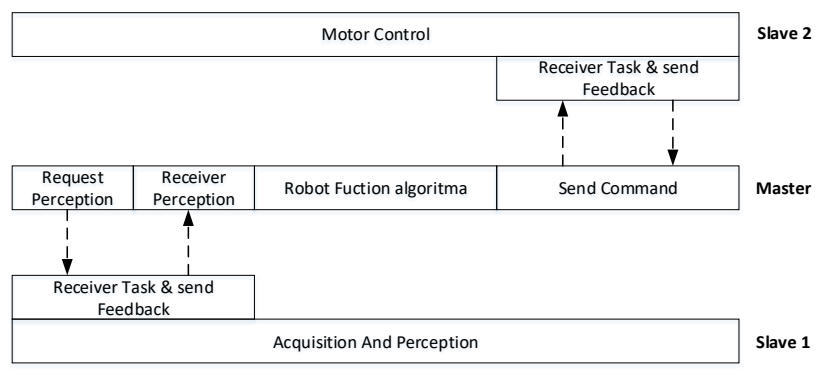

Fig. 4: Sequential process on master slaver.

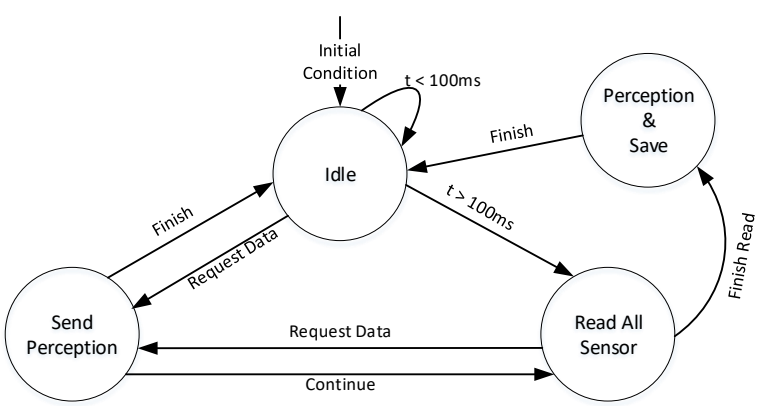

Fig. 5: State Machine Diagram of acquisition function Slave Processor

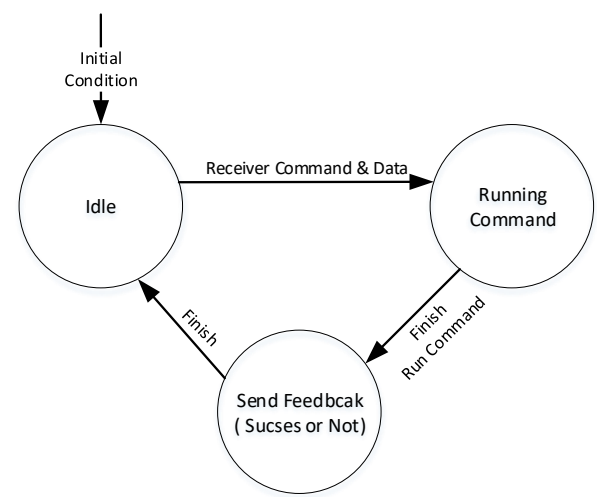

Fig. 6: State Machine Diagram of speed control functions of Slave Processor.

Both robots are tested by providing varying obstacles on two robot sensors. the maximum distance of each sensor is $90 \mathrm{~cm}$. the result of this research is the speed in performing robotic functions performed on the two embedded system models, by measuring the time required for a single process, and the number of robot functions that can run for 10 seconds.

\section{Result and Discussion}

Master microcontroller using Arduino Nano Development board, as well as slave 1 and 2 microcontrollers. This is shown in Figure 7 and figure 8 . The prototype robot has a dimension of $25 \mathrm{~cm}$ length, $20 \mathrm{~cm}$ width, and $17 \mathrm{~cm}$ high. The robot has 4 wheels with a $\mathrm{dc}$ motor drive on each wheel. But on the left wheel (front and back) connected to the same a motor driving. Likewise with the wheel on the right side. Power source robot using Lipo Battery with voltage of $7.2 \mathrm{Volt}$

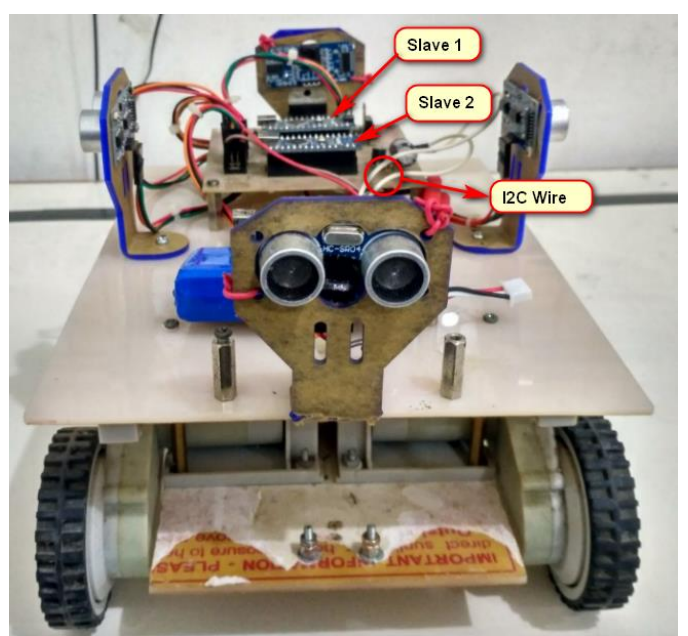

Fig. 7: Robot prototype, front view 


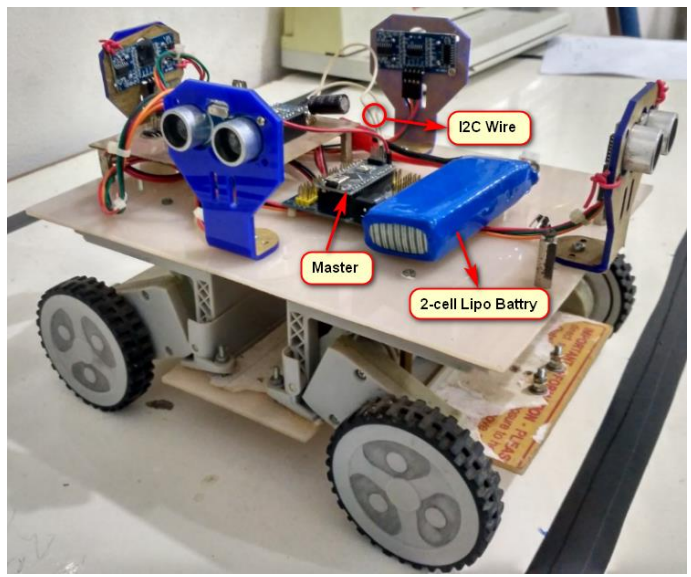

Fig. 8: Robot prototype, side view

An In the first experiment for single-processor-based systems, 2 data retrieval was performed. In the first data obtained program execution time average $66451.25 \mu \mathrm{s}$. The number of program execution iterations was obtained 150 iterations. In the second data obtained program execution time average $69204.72 \mu \mathrm{s}$. While the number of iterations 144 . The data obtained by running the robot for 10 seconds. The results of the experiments are shown in figure 9.

From both graphs obtained a maximum time of $94752 \mu \mathrm{s}$ and a minimum time of $59856 \mu$ s. The oscillations on the graph show the difference in program execution time, this is caused by the delay that occurred during the acquisition of the sensor. Thus, the program execution time is affected by the distance between the sensor and the object
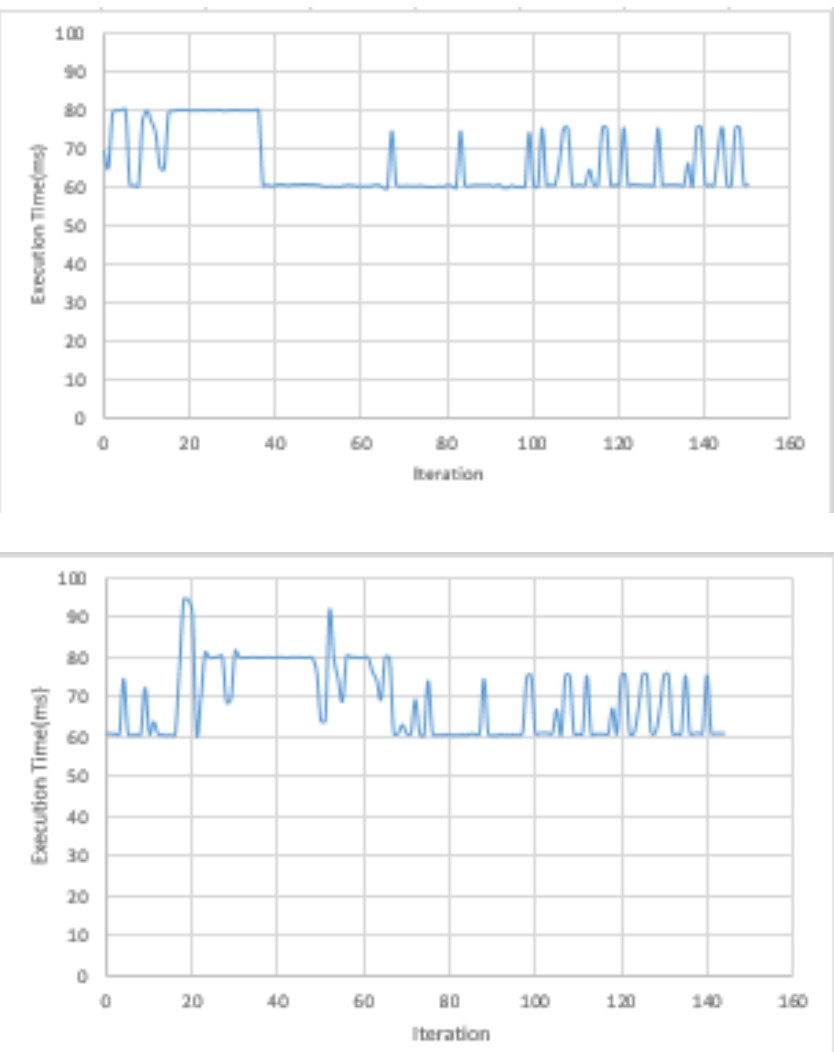

Fig. 9: Iteration and time execution in a 10s time interval on the single microcontroller, data first (top), second data (Bottom)

In the second experiment, the same way is applied to multiprocessor systems with the master-slave method. From both data got identical data. From the experimental results can be obtained the average program execution time of 546.5 us, and the number of iterations obtained 1079. Experimental results are shown in Figure 10. Oscillation does not occur in this experiment, only in the itera- tion of 0,411 and 868 increased program execution time to 1068 us. But overall there is an increase in execution time.

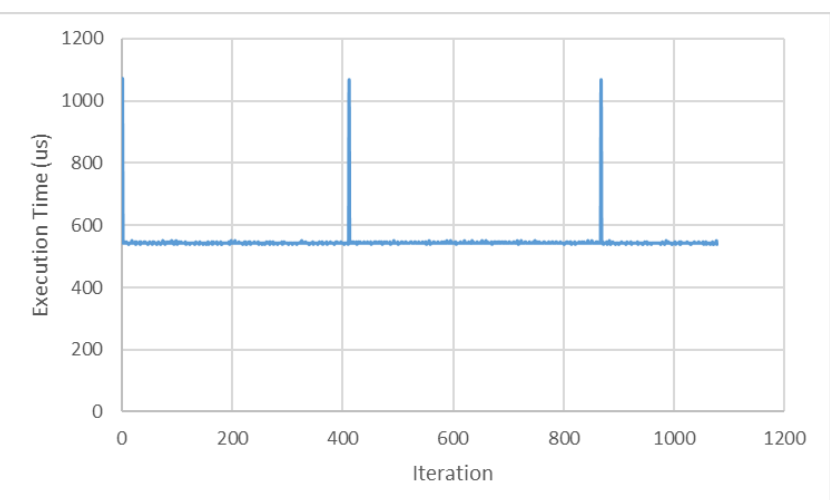

Fig. 10: Iteration and time execution in a 10s time interval on master-slave communication.

\section{Conclusion}

In From the research results can be concluded by applying multiprocessor system with master-slave method for an embedded system in a robot, have better performance with speed improvement in program execution equal to 7.3 times compared with single micro-controller system

\section{Acknowledgement}

The authors would like to thank all the members of the research group of Department of Information Technology, State Polytechnic of Samarinda.

\section{References}

[1] H. A. Alshazly and M. Hassaballah, "An Embedded System for a Bluetooth Controlled Mobile Robot Based on the ATmega8535 Microcontroller," Egyptian Computer Science Journal, vol. Volume 40, no. Issue1, pp. pp 61-72, 2016.

[2] K. Bhagat, S. Deshmukh, S. Dhonde and S. Ghag, "Obstacle Avoidance Robot," International Journal of Science, Engineering and Technology Research (IJSETR), vol. Volume 5, no. Issue 2, pp. pp 439-442, February 2016.

[3] Dr.Vasuki, "Design and Implementation of Multiprocessor Communication In Embedded Processors for Real-Time and Industrial Automation," SSRG International Journal of Electrical and Electronics Engineering (SSRG-IJEEE), pp. volume 1, Issue 1, pp 1622,2014

[4] Y. Ju, W. Paik, and M. Shin, "Design of Time Synchronization Protocol based in Master-Slave Topology for Heterogeneous USN," International Journal of Multimedia and Ubiquitous Engineering, pp. Vol 9, No 2, pp 373-384, 2014.

[5] P. Nenninger, "Design and Implementation of a Master-Slave Communication Protocol for Embedded Systems Using SystemC," in Embedded Systems, Computational Intelligence and Telematics in Control, 2012.

[6] J. Santosh and H. K., "Design Of I2C Master With Multiple Slave," International Journal on Recent and Innovation Trends in Computing and Communication, pp. Volume: 3 Issue: 5, pp 2890 - 2893, May 2015.

[7] M. Seo, H. S. Kim, J. C. Maeng, J. Kim and M. Ryu, "An Effective Design of Master-Slave Operating System Architecture for Multiprocessor Embedded Systems," in ACSAC'07 Proceedings of the 12th Asia-Pacific conference on Advances in Computer Systems Architecture, Korea, 2007.

[8] R. Siegwart and e. al, "Introduction to autonomous mobile robots, MIT press, 2011.

[9] Q. Du, L. Yan, D. Wang, Z. Ma and Y. Hu, "Design and implementation of classroom multimedia teaching equipment management system based on embedded system," in 2010 2nd International Conference on Industrial and Information Systems, Dalian, 2010. 
[10] A. Shaout, Y. Li, M. Zhou and S. Awad, "Low cost embedded weather station with intelligent system," in 10th International Computer Engineering Conference (ICENCO), Giza, 2014.

[11] S. Dalpez, A. Vaccari, R. Passerone and A. Penasa, "Design of an innovative proximity detection embedded-system for safety application in industrial machinery," in Proceedings of 2012 IEEE 17th International Conference on Emerging Technologies \& Factory Automation (ETFA), Krakow, 2012.

[12] H. Mao, Z. Hu, L. Zhu and H. Qin, "PNG File Decoding Optimization Based Embedded System," in 8th International Conference on Wireless Communications, Networking and Mobile Computing, Shanghai, 2012.
[13] J. Chhikara, R. Sinha and S. Kaila, "Implementing Communication Bridge between I2C and APB," in IEEE International Conference on Computational Intelligence \& Communication Technology, Ghaziabad, 2015.

[14] G. Petrea, V. Nicolau and M. Andrei, "Multiprocessor visual servoing system for mobile robots servicing mechatronic lines," in IEEE 23rd International Symposium for Design and Technology in Electronic Packaging (SIITME), Constanta, 2017. 